\title{
Hyperbaric oxygen enhanced mitochondria-targeted chemotherapy in bladder cancer
}

\author{
weibing $\mathrm{Li}^{1}$, chongxing Shen ${ }^{1}$, xiaofeng $\mathrm{Yue}^{1}$, linyong Dai ${ }^{1}$, jianwu Wang ${ }^{1}$, jinjin $\mathrm{Li}^{1}$, qiang \\ Fang $^{1}$, yi Zhi ${ }^{1}$, and chunmeng shi $^{2}$ \\ ${ }^{1}$ Chongqing Medical University \\ ${ }^{2}$ State Key Laboratory of Trauma, Burns and Combined Injury, Institute of Rocket Force \\ Medicine, Third Military Medical University
}

October 28, 2020

\begin{abstract}
Background and Purpose: Bladder cancer has a high rate of recurrence and drug resistance due to a lack of effective therapies. IR780 iodide, a near-infrared (NIR) mitochondria-targeting fluorescent agent, has been demonstrated to achieve higher selectivity than other drugs in different tumor types. In the study, we aimed to investigate the anti-tumor effect of IR-780 combined with hyperbaric oxygen (HBO) on bladder cancer. Experimental Approach: Using in vitro cell line data, in vivo model data and clinical data, we tested the ability of IR-780 to selectively accumulate in bladder cancer. We also evaluated the anti-tumor effect of IR-780 combined or not with HBO both in vitro and in vivo, and explored the potential mechanism of its anti-tumor effect. Key Results: We revealed for the first time that IR-780 selectively accumulated in bladder cancer (bladder cancer cells, xenografts and bladder cancer samples from patients) and could induce cancer cell apoptosis by targeting the mitochondrial complex I protein NDUFS1. Further study displayed that the combination with HBO could significantly enhance the antitumor effect of IR-780 in vitro by promoting cancer cell uptake and inducing excessive mitochondrial reactive oxygen species (ROS) production, while suppressing tumor growth and recurrence in animal models without causing apparent toxicity. Moreover, this combination antitumor strategy was also demonstrated in drug-resistant bladder cancer cells (T24/DDP) and xenografts. Conclusion and Implications: These data identify for the first time a combination of IR-780 and HBO (IR-780+HBO), which exhibits mitochondria-targeting and therapeutic capabilities, as a novel treatment paradigm for bladder cancer.
\end{abstract}

Hyperbaric oxygen enhanced mitochondria-targeted chemotherapy in bladder cancer

\section{Running title}

Hyperbaric oxygen enhanced chemotherapy in bladder cancer

Chongxing Shen ${ }^{1}$, Xiaofeng Yue ${ }^{1}$, Linyong Dai ${ }^{1}$, Jianwu Wang ${ }^{1}$, Jinjin Li $^{1}$, Qiang Fang ${ }^{1}$, Yi $\mathrm{zhi}^{1}$, Chunmeng $\mathrm{Shi}^{{ }^{*}}$ andWeibing $\mathbf{L i}^{{ }^{*}}$

${ }^{1}$ Department of Urology, The Third Affiliated Hospital (Gener Hospital) of Chongqing Medical University, Chongqing, 401120, China.

${ }^{2}$ Institute of Rocket Force Medicine, State Key Laboratory of Trauma, Burns and Combined Injury, Third Military Medical University, Chongqing, 400038, China.

Chongxing Shen and Xiaofeng Yue equally contributed to this work.

\section{Corresponding authors}


Weibing Li, Department of Urology, The Third Affiliated Hospital (Gener Hospital) of Chongqing Medical University, 401120, Chongqing, China.

E-mail: liweibing63@cqmu.edu.cn

Tel: +8613320339989

Chunmeng Shi, Institute of Rocket Force Medicine, State Key Laboratory of Trauma, Burns and Combined Injury, Third Military Medical University, 400038, Chongqing, China.

E-mail: shicm@tmmu.edu.cn

Tel: +8613708396812

\section{Acknowledgments}

This work was supported by National Natural Science Foundation of Chongqing Province (No.cstc 2018jcyjAX0034), National Key Research and Development Program (2016YFC1000805, to C. Shi), Key research incubation in the Third Affiliated Hospital of Chongqing Medical University (KY08026, to Y. Zhi).

\section{Conflict of interest}

The authors declare no competing interests.

\section{Ethics approval and consent to participate}

The collection of human tumor samples and related procedures were approved by the Ethical Committee of the Third Affiliated Hospital, Chongqing Medical University. The animal experiments in our study were conducted in accordance with the Helsinki Declaration and approved by the Laboratory Animal Welfare and Ethics Committee of Third Military Medical University.

\section{Data Availability Statement}

The data that support the findings of this study are available from the corresponding author upon reasonable request. Some data may not be made available because of privacy or ethical restrictions.

\section{Bullet point summary}

\section{-what is already known}

Bladder cancer is the most common neoplasm of the urinary tract and there are no effective therapies that delay disease recurrence or drug resistance.

IR-780, a near-infrared fluorescent agent,has been reported to have both tumor and mitochondrial targeting properties. However, research on the antitumor effect of IR-780 is limited.

\section{-What this study adds}

IR-780 induces bladder cancer cell apoptosis by targeting the electron transport chain.

Hyperbaric oxygen potentiates IR-780 antitumor efficacy in bladder cancer by promoting cancer cell uptaking and inducing excessive mitochondria reactive oxygen species.

\section{-Clinical significance}

The combination of IR-780 and hyperbaric oxygen was identified as a novel treatment paradigm for bladder cancer.

\section{Abstract}

Background and Purpose:Bladder cancer has a high rate of recurrence and drug resistance due to a lack of effective therapies. IR-780 iodide, a near-infrared (NIR) mitochondria-targeting fluorescent agent, has been demonstrated to achieve higher selectivity than other drugs in different tumor types. In the study, we 
aimed to investigate the anti-tumor effect of IR-780 combined with hyperbaric oxygen (HBO) on bladder cancer.

Experimental Approach :Using in vitro cell line data, in vivo model data and clinical data, we tested the ability of IR-780 to selectively accumulate in bladder cancer. We also evaluated the anti-tumor effect of IR-780 combined or not with HBO both in vitro and in vivo, and explored the potential mechanism of its anti-tumor effect.

Key Results: We revealed for the first time that IR-780 selectively accumulated in bladder cancer (bladder cancer cells, xenografts and bladder cancer samples from patients) and could induce cancer cell apoptosis by targeting the mitochondrial complex I protein NDUFS1. Further study displayed that the combination with HBO could significantly enhance the antitumor effect of IR-780 in vitro by promoting cancer cell uptake and inducing excessive mitochondrial reactive oxygen species (ROS) production, while suppressing tumor growth and recurrence in animal models without causing apparent toxicity. Moreover, this combination antitumor strategy was also demonstrated in drug-resistant bladder cancer cells (T24/DDP) and xenografts.

Conclusion and Implications: These data identify for the first time a combination of IR-780 and HBO (IR-780+HBO), which exhibits mitochondria-targeting and therapeutic capabilities, as a novel treatment paradigm for bladder cancer.

Keywords : Bladder cancer, drug-resistant, IR-780 dye, HBO, mitochondria-targeting

\section{Abbreviations}

NIR: near-infrared; HBO: hyperbaric oxygen; ROS: reactive oxygen species; IR-780+HBO: combination of IR-780 and HBO; NMIBC: non-muscle-invasive bladder cancer; ETC: electron transfer chain; TTFA :2-thenoyltrifluoroacetone; DPI: diphenyleneiodonium chloride; NAC: N-acetylcysteine; DDP: Cisdiamminedichloroplatinum; DOX: doxorubicin hydrochloride; FBS: fetal bovine serum; ATA: atmospheres absolute; PBS: phosphate-buffered saline; TMRM: mitochondrial membrane potential indicator; H.E: haematoxylin and eosin; FITC: fluorescein isothiocyanate; TEM: transmission electron microscopy; KRH: KrebsRinger-HEPES; MMP: mitochondrial membrane potential; T24/DDP : cisplatin-resistant T24 cancer cells; MDR: multiple drug resistance; ABC: ATP binding cassette.

\section{Introduction}

Bladder cancer is the ninth most common cancer in the world and one of the costliest cancers to manage due to the multiple therapeutic interventions needed (Svatek et al., 2014). Approximately $75 \%$ of newly diagnosed patients presents with non-muscle-invasive bladder cancer (NMIBC). Although the 5-year survival rate of NMIBC is over $80 \%$, the recurrence rate after initial treatment can reach up to $70 \%$ (Ploeg, Aben \& Kiemeney, 2009), which brings physical pain to patients and a high cost of treatment to patients' families and society. Cisplatin-based chemotherapy is currently a standard treatment for muscle-invasive and metastatic bladder cancer (Alfred Witjes et al., 2017). However, the subsequent survival benefits can only be described as modest due to chemoresistance. Despite efforts to improve surgical techniques and systemic treatment, the prognosis of bladder cancer has not changed over the past 20 years due to a lack of effective therapies (Zehnder et al., 2013), underscoring the need for new agents to reduce the recurrence of bladder cancer and drug resistance.

Mitochondria, which play a pivotal role in the regulation of tumorigenesis and progression, have recently attracted increasing attention as the subcellular target suitable for cancer therapy (WX, JD \& E, 2016). Targeting mitochondria in cancer cells may provide new strategy to tumor-targeting therapy, especially for drug-resistant cancer cells, which may be more dependent on mitochondria (JR et al., 2018; Y et al., 2019). However, this mitochondria-targeted treatment strategy is rarely studied in bladder cancer. The practical application of current mitochondrial-targeted therapy is limited due to the poor tumor-specific distribution and reduced drug accumulation in mitochondria. Recently, a couple of near infrared heptamethine dyes with both tumor and mitochondrial targeting have attracted much attention. IR-780 iodide, a near-infrared (NIR) fluorescent agent, has been reported to achieve higher selectivity than other drugs for the mitochondria of 
different types of tumor cells and xenografts (Zhang et al., 2010). In addition, IR-780 has an absorption peak at $780 \mathrm{~nm}$ and can emit fluorescence with a high intensity in the 807-823 nm wavelength range, which facilitates its use for imaging applications (Kuang et al., 2017). However, research on the anti-tumor effect and mechanism of IR-780 is limited. In the current study, we revealed for the first time that IR-780 selectively accumulated in bladder cancer and can induce cancer cell apoptosis by targeting the mitochondrial electron transfer chain (ETC). Further research found that combined treatment with IR-780 and HBO exerted an incredible antitumor effect, representing a novel candidate strategy for bladder cancer-targeted therapy.

\section{Materials andMethods}

\section{Materials}

IR-780 iodide, 2-thenoyltrifluoroacetone (TTFA), diphenyleneiodonium chloride (DPI), $\mathrm{H}_{2} \mathrm{O}_{2}$, and $\mathrm{N}$-acetylcysteine (NAC) were obtained from Sigma-Aldrich (St. Louis, MO, USA). Cisdiamminedichloroplatinum (DDP) and doxorubicin hydrochloride (DOX) were obtained from Aladdin (Shanghai, China). MitoTracker Green, mitoSOX, and the mitochondrial membrane potential indicator TMRM were purchased from Invitrogen (Carlsbad, CA, USA). A Complex I Enzyme Activity Microplate Assay was purchased from Abcam (Cambridge, MA, USA). DiBAC4(3) was purchased from MedChem Express (Monmouth Junction, NJ, USA). A Reactive Oxygen Species (ROS) Assay Kit, Hoechst 33258, Crystal Violet Staining Solution, and an ATP Assay Kit were obtained from Beyotime (Shanghai, China). Primary antibodies were Rb anti-Cytochrome C (1:1000, CST, 11940T), Rb anti-Ndufs1 (1:1000, abcam, ab169540), Rb anti-Caspase-9 (1:1000, abcam, ab202068), Rb anti-Caspase 3 Antibody (1:1000, proteintech, 19677-1AP), Rb anti-cleaved Caspase 3 Antibody (1:1000, abcam, ab13847), Rb anti-PARP (1:1000, proteintech, 13371-1-AP) and Ms anti- $\beta$-action (1:5000, proteintech, 6008-1-Ig).

\section{Cell lines}

Human bladder cell lines (T24, 5637, and TCCSUP) were purchased from the ATCC (Manassas, VA,

USA), and human normal cell lines (HK2 and SV-HUC-1) were obtained from the Chinese Academy of Sciences Cell Bank (Shanghai, China). A mouse bladder cell line (MB49) was donated by Dr. Chunmeng Shi from the Institute of Rocket Force Medicine, Third Military Medical University (Chongqing, China). All cells were cultured in their recommended medium supplemented with $10 \%$ FBS (Gibco, USA) and 1\% penicillin/streptomycin (Beyotime). All cells were passaged at 1:3 ratios and cultured in an incubator at 37 ${ }^{\circ} \mathrm{C}$ with $5 \% \mathrm{CO}_{2}$. T24/DDP, a cisplatin-resistant bladder cancer cell line, was established by a concentration gradient method.

\section{Methods \\ HBO exposure}

An HBO exposure experiment was performed in a temperature-adjustable hyperbaric chamber provided by the neurosurgery department of Southwest Hospital (Chongqing, China). The main parameters for HBO administration were 2.5 atmospheres absolute (ATA) of pure oxygen and a time of $120 \mathrm{~min}$, and the program included $15 \mathrm{~min}$ of compression and decompression at a constant velocity. The temperature for cells was 37 ${ }^{\circ} \mathrm{C}$, while that for animals was $25{ }^{\circ} \mathrm{C}$. Cells in different plates were removed from the chamber after $\mathrm{HBO}$ exposure and cultured routinely in an incubator.

\section{Tumor models}

MB49 cancer cells grown to $70 \%$ confluence were suspended in phosphate-buffered saline (PBS). MB49 transplanting tumor model were established in female C57 BL/6 mice (6-8 weeks old and weighing 17-20 g) by subcutaneously implanting each mouse with $1^{*} 10^{7}$ cells. T24, T24/DDP tumor xenografts were established in female nude mice in the same way.

\section{Optical imaging}


In vivo fluorescence imaging mice bearing T24, MB49 transplanted tumors were sacrificed at $24 \mathrm{~h}$ ( $\mathrm{n}=5$ each) after intraperitoneal injection with IR-780 at a dose of $1 \mathrm{mg} / \mathrm{kg}$. The dissected organs were subjected to NIR imaging with a Kodak In-Vivo FX Professional Imaging System (New Haven, CT). Cellular dye uptake and the subcellular localization cells of different cell lines (T24, MB49, HK2, and SV-HUC-1) were seeded at the same densities in culture dishes and cultured for $24 \mathrm{~h}$. Then, the cells were incubated with $2 \mu \mathrm{M}$ IR-780 in 1640 culture medium for $15 \mathrm{~min}$ in the incubator before being incubated for 10 min with $1 \mu \mathrm{M}$ MitoTracker Green. Hoechst was used to stain the nuclei. Finally, these cells were imaged under a confocal microscope.

\section{Cell viability evaluation}

Bladder cancer cell lines of different grades (T24, 5637, TCCSUP, T24/DDP and MB49) were treated with the indicated doses of IR-780 with or without HBO exposure, and a CCK-8 kit (Dojindo Kumamoto, Japan) was used to measure cell proliferation after $48 \mathrm{~h}$. T24 cells after treatment with or without different inhibitors were placed in $7.5 \mu \mathrm{M}$ IR-780+HBO, and cell viability was detected after $48 \mathrm{~h}$.

\section{In vivo IR-780+HBO evaluation}

In vivo, treatment was administered on the fifth day of transplanted tumor establishment through intraperitoneal injection of $3 \mathrm{mg} / \mathrm{kg}$ IR-780. The IR-780+HBO group and the HBO-only group were then placed in the hyperbaric chamber $4 \mathrm{~h}$ after IR-780 injection. The change in tumor volume was monitored every other day during the experiment. After treatment every two days for a total of 5 interventions, the mice were weighed and sacrificed, and the transplanted tumors from the animals were photographed and weighed. Subsequently, the tumor tissues and organs were dissected and fixed in $4 \%$ paraformaldehyde. Hematoxylin and eosin (H.E.) staining was performed to observe the toxicity in normal tissues. To study the long-term development of tumors after IR-780+HBO treatment, tumor volume was monitored in the IR-780+HBO group and DDP group. After 5 injections of $3 \mathrm{mg} / \mathrm{kg}$ IR-780 and $3 \mathrm{mg} / \mathrm{kg}$ DDP, the mice continued to be fed for 28 days. To compare the anti-tumor effect of IR-780+HBO and DDP on drug-resistant bladder cancer, $3 \mathrm{mg} / \mathrm{kg}$ IR-780 and $3 \mathrm{mg} / \mathrm{kg}$ DDP were injected into transplanted tumor mice in the IR-780 group and DDP group respectively. After treatment every two days for a total of 5 interventions, the mice were photographed and sacrificed, and the transplanted tumor from the animals were photographed and weighed.

\section{Flow cytometry}

For the apoptosis assay, cells were collected after treatment, and an Annexin V/PI detection kit (BD Biosciences) was used to detect apoptosis by flow cytometry according to the protocol described. For the ROS assay, cells were incubated with the probe DCFH-DA at a concentration of $10 \mu \mathrm{M}$ after the different treatments for $30 \mathrm{~min}$ at $37^{\circ} \mathrm{C}$, and then flow cytometry was used for fluorescence intensity detection. For the mitochondrial ROS assay, cells subjected to the different treatments were incubated with the probe mitoSOX at a concentration of $5 \mu \mathrm{M}$ for $10 \mathrm{~min}$ at $37^{\circ} \mathrm{C}$, and then flow cytometry was used for fluorescence intensity detection. For the plasma membrane potential assay, cells were incubated in KRH (Krebs-Ringer-HEPES) buffer containing $100 \mathrm{nM}$ DiBAC4(3) for 20 min at room temperature before flow cytometry detection. For the mitochondrial membrane potential (MMP) assay, TMRM was also detected by flow cytometry.

\section{Colony formation assay}

After intervention for $6 \mathrm{~h}$, cells were seeded in six-well plates at a density of 1000 cells per well and cultured overnight to attach. The medium was replaced with fresh medium after $24 \mathrm{~h}$, and the cells were cultured for 12 days. Subsequently, the cells were fixed with paraformaldehyde for $10 \mathrm{~min}$ and subjected to crystal violet (Beyotime) staining for $5 \mathrm{~min}$. The samples were photographed, and the colonies $>0.2 \mathrm{~mm}$ in diameter were counted under a microscope.

\section{Transmission electron microscopy (TEM)}

After $24 \mathrm{~h}$ of treatment, cells were collected and fixed overnight at $4{ }^{\circ} \mathrm{C}$ in $2.5 \%$ glutaraldehyde. Then, the cells were subjected to secondary fixation in $2 \%$ osmium tetroxide followed by embedding in resin. Images of thin sections were obtained by TEM. 


\section{Western blot analysis}

Cells were harvested after treatment for $24 \mathrm{~h}$, and total proteins were extracted with RIPA lysis buffer (Beyotime). After separation using 12\% SDS-polyacrylamide gels, the proteins were transferred onto PVDF membranes (Millipore, USA). The PVDF membranes were blocked with Quick Blocking Buffer (Beyotime) for $15 \mathrm{~min}$ and then incubated overnight with primary antibodies at $4{ }^{\circ} \mathrm{C}$. The antibodies described above were used. After incubation for $1 \mathrm{~h}$ with secondary antibodies in an incubator at $37^{\circ} \mathrm{C}$, the signals were identified by chemiluminescence detection.

\section{Statistical analysis}

All data are expressed as the means \pm standard derivation. Statistical analysis of experimental data was performed using GraphPad Prism 8.0. Comparisons between two groups were performed using the student' t-test, one-way analysis of variance (ANOVA) or two-way ANOVA were used for multiple groups. $P$-values $<0.05$ were considered to indicate statistical significance.

\section{Results}

\section{IR-780 selectively accumulates in the mitochondria of bladder cancer cells}

The ability of IR-780 to selectively accumulate in bladder tumors was studied with T24 and MB49 transplanted tumors. The organs of nude mice with pre-established T24 tumor xenografts and C57 BL/6 mice with pre-established MB49 transplanted tumor were imaged after intraperitoneal injection of $1 \mathrm{mg} / \mathrm{kg}$ IR-780. The fluorescence distribution of the dissected organs shown in Fig. 1A and B confirmed the preferential aggregation of IR-780 in tumors at $24 \mathrm{~h}$ after injection. To further clarify the time course of IR-780 distribution in tumor-bearing mice, the dissected organs were imaged at different times after IR-780 injection. Fig. S1 revealed that the accumulation of IR-780 in tumors gradually increased with time, reaching its peak at $4 \mathrm{~h}$, while less uptake occurred in other (normal) tissues. Moreover, IR-780 preferentially accumulated in bladder tumor tissues from clinical samples (Fig. 1C and D), indicating that the NIR small-molecule dye IR-780 had the ability to target bladder tumors. Subcellular uptake and localization analysis of IR-780 through assessment of IR-780 and MitoTracker Green colocalization in T24 and MB49 cells revealed that IR-780 exclusively accumulated in the mitochondria of bladder cancer cells (Fig. 1E); HK2 and SV-HUC-1 cells were used as controls.

\section{IR-780 induces cancer cell apoptosis bytargeting the mitochondrial electron transport chain}

Since IR-780 has the ability to target bladder cancer cell, its antitumor effect should be investigated. Fig.2A shows that IR-780 could inhibit bladder cancer cells (T24, 5637, TCCSUP, MB49) proliferation in a dosedependent manner $(\mathrm{p}<0.05)$. The apoptosis rate of T24 cells after incubation with $15 \mu \mathrm{M}$ IR-780 was more than $30 \%$ compared with $3 \%$ in the control group (Fig. $2 \mathrm{~B} ; \mathrm{p}<0.05$ ), suggesting that IR-780 could induce T24 cells apoptosis in a does-dependent manner. As we confirmed that IR-780 specifically accumulated in mitochondria, the influence on mitochondrial function should be studied. Fig. $2 \mathrm{C}$ demonstrates that IR-780 markedly increased mitochondrial ROS, reduced cellular ATP production (Fig. 2D) and decreased mitochondrial membrane potential (Fig. 2E) in a dose-dependent manner, which could activate apoptosis signaling pathways and induce cancer cell apoptosis. In addition, IR-780 significantly inhibited mitochondrial complex I activity (Fig. 2F) and reduced the expression of complex I subunit protein NDUFS1 (Fig. 2G) in T24 cells in a dose-dependent manner, consistent with the findings of our previous study confirming IR-34 (a NIR fluorescent dye) targets and cleaves the mitochondrial complex I protein NDUFS1 in cancer cells(Wang et al., 2018). Clearance of the NDUFS1 protein disturbed electron transport in the ETC, which led to considerable electronic leakage, promoting mitochondrial ROS production.

\section{HBO promotes IR-780 antitumorefficacy in vitro}

To investigate the antitumor activity of IR-780+HBO in vitro, bladder cancer cell lines of different grades (T24, 5637, TCCSUP, and MB49) were incubated with the indicated doses of IR-780 combined or not with HBO. Cell viability was measured with a CCK-8 kit. Fig. 3A shows that IR-780+HBO inhibited cancer cell 
proliferation in a dose-dependent manner and displayed better anticancer activity than IR-780 alone. The $7.5 \mu \mathrm{M}$ IR-780 + HBO treatment almost achieved the efficacy of the $15 \mu \mathrm{M}$ IR-780 treatment in T24 cells and had little effect on normal cells (Fig. S2A). T24 cells were treated with DOX or DDP with or without HBO to further investigate whether this combination strategy also applied to other chemotherapeutic drugs. As shown in Fig. S2B, there was no significant difference between drugs (DOX and DDP) combined or not with HBO. Since bladder cancer cells have the ability to self-renew, we first investigated the effect of IR-780+HBO on this characteristic. Compared with the other groups, the group treated with $7.5 \mu \mathrm{M}$ IR-780 combined with HBO significantly inhibited the colony formation ability of T24 cancer cells (Fig. 3B and C). The cells were obviously wrinkled and rounded upon microscopic examination $16 \mathrm{~h}$ after treatment with 7.5 $\mu \mathrm{M}$ IR-780 +HBO (Fig. 3F), which suggested that cell death may have been the main cause of the decrease in cell numbers. As shown in Fig. 3D and E, the apoptosis rate in the $7.5 \mu \mathrm{M}$ IR-780+HBO group was significantly higher than in the other groups. Moreover, we found that the levels of Cytochrome $\mathrm{C}$ and the apoptosis markers PARP, caspase3, and cleaved caspase9 (c-caspase9) were all increased by IR-780+HBO (Fig. 3G). The apoptosis inhibitor z-VAD-FMK partly rescued cell death, increasing cell viability (Fig. 3H). All these results suggested that HBO could enhance IR-780 antitumor efficacy by inducing classic apoptosis in T24 cells.

\section{The antitumor efficacy of IR-780+HBO in vivo}

Based on the excellent antitumor effect of IR-780+HBO in vitro, we further studied its anti-tumor effect in vivo. MB49 tumor-bearing mice were injected intraperitoneally with IR-780 combined or not with HBO every two days for a total of 5 injections. Analysis of the volumes of the tumors in the different intervention groups showed that the IR-780+HBO group exhibited the best tumor inhibition among the groups (Fig. 4A). The HBO-only treatment had no effect on tumor growth, and compared with the control, IR-780 alone exerted a modest tumor-inhibiting effect. The sizes and shapes of the tumors were photographed (Fig. 4B), and tumor weight was detected (Fig. 4C); these results also suggested that IR-780+HBO had a stronger ability to inhibit tumor growth than the other treatments. The body weights among the groups did not significantly differ (Fig. 4D), and abnormal histopathological changes were not observed in normal tissues (Fig. 4G), which indicated that IR-780+HBO treatment did not cause obvious side effects. Since bladder cancer has a high rate of recurrence, we further investigated whether IR-780+HBO could delay tumor recurrence. We observed the growth of tumors for a longer time period and compared the effects of IR-780+HBO with those of the classic antitumor drug cisplatin. Interestingly, the volumes of the tumors in the mice subjected to IR-780+HBO therapy did not change significantly, while those of the tumors in mice subjected to DDP therapy continued to increase after intervention (Fig. 4E). On day 28, the tumors from mice treated with IR-780+HBO were much smaller than those from mice treated with DDP (Fig. 4F). These studies indicated that IR-780+HBO could effectively inhibit the growth of tumors and delay tumor recurrence in vivo without causing obvious side effects.

\section{HBO promotesuptake of IR-780 in cancer cells byincreasingplasma}

\section{membrane potential}

We have confirmed that IR-780 alone can induce apoptosis in cancer cells in a dose-dependent manner. To test whether HBO promotes the absorption of IR-780, the uptake of IR-780 was compared in T24 cells with different interventions. As shown in Fig. $5 \mathrm{~A}$ and $\mathrm{B}$, the fluorescence intensity was obviously enhanced in the group treated with combined IR-780 and HBO compared with the IR-780-only group. In addition, HBO did not change the fluorescence properties of IR-780 (Fig. 5C), and the levels of NDUFS1, the complex I subunit protein, were decreased in the $7.5 \mu \mathrm{M}$ IR-780 + HBO group compared with the IR-780-only group (Fig. 5D), further confirming that HBO enhanced the accumulation of IR-780. Our previous study has shown that the plasma membrane potential plays a critical role in the uptake of IR-780 in cancer cells(Zhang, Luo, Tan \& Shi, 2014). Therefore, plasma membrane potential was measured using the voltage-dependent fluorescent oxonol dye DiBAC4(3) in T24 cells after pre-IR-780 (IR-780 was removed after incubation for 30 min) treatment combined or not with HBO treatment. DiBAC4(3) was a lipophilic anionic fluorescent dye, which could enter cells through depolarized plasma membrane and enhance fluorescence intensity. Fig. 5E revealed that 
the fluorescence intensity of DiBAC4(3) in T24 cells was significantly decreased in the pre-IR-780+ HBO group, indicating hyperpolarization of plasma membrane, and the plasma membrane potential was elevated. To further clarify the principal role of plasma membrane potential in the accumulation of IR-780 in T24 cells, cells were pre-incubated for $1 \mathrm{~h}$ with different doses of $\mathrm{K}^{+}$medium to depolarize the plasma membrane (Fig. 5F) and then incubated with $2 \mu \mathrm{M}$ IR-780 for $10 \mathrm{~min}$. As shown in Fig. 5G and H, the fluorescence intensity of IR-780 decreased gradually with the increase in $\mathrm{K}^{+}$concentration. Moreover, after $2 \mu \mathrm{M}$ IR780 treated for $30 \mathrm{~min}$, T24 cells were incubated with different does of $\mathrm{K}^{+}$medium and then treated with HBO. The fluorescence intensity revealed that the absorption enhancement of IR-780 caused by HBO was gradually weakened with the increase in $\mathrm{K}^{+}$concentration (Fig. 5I). Taken together, these studies strongly suggested that HBO promoted the accumulation of IR-780 in T24 cells by changing the plasma membrane potential. We ascertained that high doses of IR-780 could lead to cell death in our previous study, but we wondered whether the enhanced uptake of IR-780 was the primary cause of cell death during IR-780+HBO treatment. Therefore, we first validated that the fluorescence intensity of IR-780 reached its peak at 30 min when cells were incubated with $7.5 \mu \mathrm{M}$ IR-780 (Fig. S3A). Second, we compared the fluorescence intensity between cells incubated with IR-780 and cells pre-incubated with IR-780 at different times. As shown in Fig.S3B, there were no significant differences in fluorescence intensity between the two groups at corresponding times. Finally, T24 cells were incubated with $7.5 \mu \mathrm{M}$ IR-780 or pre-IR-780 and combined or not with HBO treatment. As shown in Fig. 5J, pre-IR-780+HBO displayed better tumor inhibition than IR-780 alone; however, pre-IR-780+HBO was not as effective as IR-780+HBO. These results indicated that the enhanced uptake of IR-780 was one of the causes of the antitumor effects of IR-780+HBO.

\section{HBOenhances IR-780 antitumor efficacy by inducing excessivemitochondrial ROS}

To further explore the main mechanism of anti-tumor effect of IR-780+HBO, ROS production was measured with the probe DCFH-DA in T24 cells. Fig. 6A and B revealed that ROS production in T24 cells was significantly enhanced in the IR-780+ HBO group. Given that IR-780 localizes to mitochondria, we tested mitochondrial ROS production with the probe mitoSOX. Fig. 6C and D show that the mitoSOX fluorescence levels in T24 cells rapidly increased in the IR-780+HBO group immediately after HBO treatment. The elevations in mitoSOX fluorescence were positively associated with cell death (Fig. 6E). Treatment with NAC and MitoQ (a mitochondrial ROS inhibitor) before treatment with IR-780+HBO could obviously restrain the increases in mitoSOX levels, and increasing the survival rates of T24 cells also decreased the expression of the apoptosis marker c-caspase3, but this effect was limited by diphenyleneiodonium (DPI; an NADPH oxidase inhibitor) (Fig. 6F-H), further confirming that IR-780+HBO-induced ROS was mainly from mitochondria. Moreover, T24 cells incubated with or without $7.5 \mu \mathrm{M}$ IR-780 for 30 min were treated with different doses of $\mathrm{H}_{2} \mathrm{O}_{2}$ for $48 \mathrm{~h}$, and the apoptosis rate of the IR-780+ $\mathrm{H}_{2} \mathrm{O}_{2}$ group was significantly higher than the control group (Fig. 6I). These studies indicated that mitochondrial ROS played a critical role in IR-780+HBO-induced cell death. The antitumor effects of IR-780+HBO could be eliminated when the oxygen concentration was reduced to $21 \%$ (Fig. S4A). However, with the increased oxygen concentration, the cell survival rate gradually decreased (Fig. S4B), which suggested that oxygen, rather than pressure, played an important role in the antitumor effect. To further explore the mechanism by which IR-780+HBO increased mitochondrial ROS, the concentration of oxygen was changed during HBO treatment, and TTFA (a mitochondrial complex II inhibitor), which can inhibit back-propagation of electrons, was used before IR-780+HBO treatment. mitoSOX fluorescence decreased with decreasing oxygen concentrations (Fig. 6J), and TTFA could partially reduce mitoSOX fluorescence (Fig. 6K), suggesting that oxygen and electrons are the crucial factors mediating the increased mitochondrial ROS induced by IR-780+HBO. Furthermore, mitochondrial membrane potential, which plays an important role in regulating apoptosis, decreased rapidly when T24 cells were treated with IR-780 for 30 min (Fig. S5A). Further research revealed that when the concentration of IR-780 was $7.5 \mu \mathrm{M}$, the mitochondrial membrane potential was decreased at 30 min but increased with time and returned to normal by $24 \mathrm{~h}$ (Fig. S4B). However, $7.5 \mu \mathrm{M}$ IR-780 combined with HBO further reduced the mitochondrial membrane potential (Fig. 6L), which could aggravate the damage to mitochondria. As shown by TEM imaging (Fig. 6M), mitochondrial vacuolation was induced by IR$780+$ HBO treatment, which was closely related to cell death. 


\section{The effects of IR-780+HBO onDDP-resistant T24 cells}

Cisplatin resistance is an important barrier in bladder cancer treatment that has a close relationship with prognosis. Previous studies have demonstrated higher selective accumulation of IR-780 than other drugs in drug-resistant lung cancer cells. To verify whether this accumulation advantage occurs in cisplatinresistant bladder cancer cells, we explored the accumulation of IR-780 in cisplatin-resistant T24 cancer cells (T24/DDP). As shown in Fig. 7A and B, the fluorescence intensity in T24/DDP cells was stronger than in T24 cells. IR-780 was intraperitoneally injected into athymic nude mice bearing subcutaneous T24/DDP cell xenografts to further validate the accumulation ability in vivo. The tumor xenografts were clearly demarcated in these mice $24 \mathrm{~h}$ after IR-780 injection (Fig. 7C). Next, we validated the anticancer effect of IR-780+HBO on T24/DDP cells. As shown in Fig. 7E, IR-780+HBO inhibited T24/DDP cell proliferation in a dose-dependent manner and exhibited better anticancer activity than DDP and IR-780 alone. In vivo, T24/DDP tumor-bearing nude mice were injected intraperitoneally with IR-780 (combined with HBO) or DDP; the treatment was administered every two days for a total of 5 treatments. IR-780+HBO showed a more pronounced tumor-inhibiting effect than the other treatments (Fig. 7F and G). Interestingly, we found that some tumor xenografts $(4 / 7)$ in the IR-780+HBO group appeared to be ruptured (Fig. $7 \mathrm{H})$, which may have been due to the rapid excessive production of ROS.

\section{Discussion}

The present treatments for bladder cancer are unsatisfactory in terms of the treatment methods, therapeutic effects and quality of life of the patients, due to the lack of effective methods to reduce the recurrence of bladder cancer and drug resistance. Even with the advent of neoadjuvant therapy and immunotherapy, there was no significant change in prognosis. Therefore, there are still obvious unmet medical needs, and more treatments are needed. IR-780, a mitochondria-targeted fluorescent small molecule, has been confirmed to delay tumor recurrence in a lung tumor model(Wang, Liu, Zhang, Luo, Tan \& Shi, 2014). In the current study, we investigated the effect of IR-780 in bladder cancer. It was confirmed that IR-780 could preferentially accumulate in the mitochondria of bladder cancer cells. Further studies have shown that IR780 rapidly targets the mitochondrial complex I protein NDUFS1 of cancer cells, resulting in a large number of electron leakage for ROS production and can induce cancer cell apoptosis. Moreover, IR-780 combined with HBO could significantly enhanced the antitumor effect of IR-780 both in vitro and vivo, indicating that the combination of IR-780 and HBO could be a therapeutic strategy for bladder cancer.

Mitochondria in cancer cells are essentially intact and play key roles in energy production and apoptotic pathways(Jose, Bellance \& Rossignol, 2011; Xiao, Fan, Huang, Gu, Li \& Liu, 2010). Increasing evidence indicates that mitochondrial biosynthesis, bioenergetics and signaling are essential for tumorigenesis. Hence, mitochondria have been considered as subcellular targets for tumor targeting and therapy (Weinberg \& Chandel, 2015; Wen, Zhu \& Huang, 2013). In particular, as increased levels of ROS and altered redox statuses have been observed in cancer cells(Berkenblit et al., 2007), mitochondria in cancer cells are believed to be more susceptible to increased ROS than those in normal cells. Dysfunctional mitochondria are essential sources of ROS, which are produced by leakage of the ETC, and can activate intrinsic apoptosis(Porporato, Filigheddu, Pedro, Kroemer \& Galluzzi, 2017; Sabharwal \& Schumacker, 2014). Evidence shows that most cancer cells have normal mitochondrial respiratory function, and some cancers even show a more active ETC than normal cells because of the rapid turnover of cancer cells(Deribe et al., 2018). Inhibition of the ETC has been shown to exert antitumor effects in different types of cancers (Hao, Chang, Tsao \& Xu, 2010; Roesch et al., 2013). However, the poor tumor targeting of these drugs limits their further clinical application. Recently, a near-infrared fluorescent dye, IR-780, which has both mitochondrial and tumor targeting, has been widely observed and researched. Current strategies for IR-780 mainly involve nanotechnology or multistep chemical coupling of numerous functional agents, including tumor-specific ligands and antitumor drugs(Cao et al., 2017; Uthaman et al., 2018; Yan et al., 2016). Although these strategies have revealed some antitumor effects, there are still great challenges hindering their further application, such as adverse immunogenic reactions, inefficient drug delivery systems and difficulties in mass production (Desai, 2012). Very recently,

a number of photosensitizers for mitochondrial targets have been designed and administered to absorb light 
energy and used to induce excessive ROS production, leading to cancer cell death(Luo et al., 2016; Tan et al., 2017). However, though NIR excitation has better tissue penetration than ultraviolet excitation (Idris, Jayakumar, Bansal \& Zhang, 2015; Lucky, Muhammad Idris, Li, Huang, Soo \& Zhang, 2015), it is still difficult to apply it to internal organs in the human body because the NIR excitation cannot reach these organs. In this study, we chose oxygen as a sensitizer of IR-780, as oxygen can be transported to various tissues in the body during HBO treatment. We confirmed that IR-780 could preferentially accumulate in the mitochondria of bladder cancer cells and disturb electron transport in the ETC by targeting the complex I subunit protein NDUFS1, which caused many electrons to leak out and promote ROS production. The mitoSOX fluorescence in T24 cells increased gradually and reached its peak at $3 \mathrm{~h}$ upon treatment with 7.5 $\mu \mathrm{M}$ IR-780 alone (Fig. S5C). However, when IR-780 was combined with HBO, mitoSOX fluorescence at $3 \mathrm{~h}$ was more than twice as high as that after IR-780 treatment alone (Fig. 6D), which could have been the result of a combination of enough oxygen and leaked electrons. Moreover, we found that IR-780 rapidly reduced the mitochondrial membrane potential, which may have been related to its rapid entry into mitochondria. The mitochondrial membrane potential gradually recovered with time after treatment with $7.5 \mu \mathrm{M}$ IR-780 alone. However, the mitochondrial membrane potential remained at low levels during the $2 \mathrm{~h}$ of combined treatment with HBO, which made the mitochondria more vulnerable to increased ROS levels and led to apoptosis through the mitochondrial pathway.

Hypoxia, a common characteristic of most solid tumors, remains a significant barrier to therapeutic efficacy. Therefore, various strategies for increasing oxygen tension in hypoxic solid tumors have been urgently pursued(Dewhirst, Mowery, Mitchell, Cherukuri \& Secomb, 2019). HBO, which is used as an adjuvant treatment with chemotherapy, has been demonstrated to significantly improve the efficacy of various chemotherapeutic drugs(Stepień, Ostrowski \& Matyja, 2016). Alteration of the hypoxic environment of tumors, promotion of the absorption of drugs in tumors, and enhancement of the sensitivity of tumors to drugs are considered to be the main mechanisms by which HBO sensitizes cells to chemotherapy. However, HBO may aggravate the toxicity of some drugs (DDP and DOX) toward normal tissues(Selvendiran et al., 2010), which limits its further application. In this study, we found that HBO, when combined with IR-780, affected the antitumor process not only by increasing the killing capacity of the drug itself but also by providing the necessary oxygen support for the explosive production of ROS (as indicated by mitoSOX fluorescence), which could have been the main cause of cell death. The existence of hypoxia in bladder tumor has been confirmed(Hoskin, Sibtain, Daley \& Wilson, 2003), which will seriously affect the ROS production after IR-780 enters the tumor. However, the combination with HBO could compensate for this defect and maximize the anti-tumor effect of IR-780. In addition, our study also indicated that IR-780+HBO did not produce significant toxicity in normal tissues, which may have been related to the strong tumor-targeting ability of IR-780.

The mechanism by which IR-780 iodide selectively accumulates in tumor cell has been investigated in our previous research, which indicated that plasma membrane potential plays a critical role in IR-780 iodide uptake in tumor cells(Zhang, Luo, Tan \& Shi, 2014). IR- 780 iodide is a lipophilic cation, and some researchers have confirmed that in normal and carcinoma cells, the difference in accumulation of lipophilic cation can be directly attributed to the difference in membrane potential(Davis, Weiss, Wong, Lampidis \& Chen, 1985). It is also have been reported that the accumulation of any charged species across the membrane is determined by the membrane potential(Zielonka et al., 2017). Our data indicated that the absorption of IR-780 in bladder cancer cells was significantly reduced after the plasma membrane was depolarized by increasing the concentration of $\mathrm{K}^{+}$in cell culture medium, which further confirmed the critical role of plasma membrane potential for IR-780 iodide uptake in bladder cancer. Interestingly, we found that HBO enhanced the uptake of IR-780 in bladder cancer cells in a plasma membrane potential-mediated manner. Inhibiting the plasma membrane potential with a certain concentration of $\mathrm{K}^{+}$could reduce the absorption of IR-780 caused by HBO. However, the mechanism by which HBO altered the plasma membrane potential of cells pretreated with IR-780 is not well understood. Increased ROS has been reported to cause the changes in plasma membrane potential by hyperpolarizing the plasma membrane in macrophages(Klyubin, Kirpichnikova, Ischenko, Zhakhov \& Gamaley, 2000). In the current study, our data showed that HBO increased the ROS production in bladder cancer cells pretreated with IR-780, and ROS production induced by 
$\mathrm{H}_{2} \mathrm{O}_{2}$ could change the plasma membrane potential of bladder cancer cell, resulting in enhanced absorption of IR-780 (Fig. S6). The plasma membrane potential of epithelial cells is considered to be primarily a potassium diffusion potential. Therefore, we speculate that the elevated ROS during HBO may cause intracellular K + to flow out, leading to changes in plasma membrane potential. However, the specific mechanism requires further exploration.

Drug resistance is one of the main causes of recurrence after bladder cancer radiotherapy and chemotherapy. The mechanisms related to drug resistance include reduced drug accumulation in cancer cells, increased antiapoptotic ability, and overexpression of certain multiple drug resistance (MDR) proteins, such as the ATP binding cassette (ABC) transporter(Housman et al., 2014). However, most antidrug behavior is closely related to mitochondria. Targeting of mitochondria and modulation of ROS have been shown to be an effective strategy against different types of drug-resistant cancer cells(Cui et al., 2018). Alexander Roesch (A et al., 2013)confirmed that restraint of the ETC overcomes multidrug resistance in melanoma and revealed long-term effects. Elesclomol, an ETC-targeting compound, can cause mitochondrial ROS by disrupting the ETC and causing the death of cisplatin-resistant melanoma cells (Cierlitza et al., 2015; Santos et al., 2012). Rotenone, an ETC complex I inhibitor, can increase ROS production and induce apoptosis in DOX-resistant cancer cells(Wu et al., 2018). However, to the best of our knowledge, studies investigating mitochondrial targeting in drug-resistant bladder cancer are limited. In the current study, we demonstrated that IR780 could target the oxidative respiratory chain of bladder cancer cells and accumulate to greater levels in T24/DDP than T24 cells. When combined with HBO, it could obviously promote apoptosis of T24/DDP cells, suggesting a new therapeutic strategy for DDP-resistant bladder cancer.

\section{Conclusions}

In this study, we identified a mitochondria-targeted fluorescent small molecule, IR-780, which can selectively target the mitochondria of bladder cancer cells, including cisplatin-resistant cells, and induce cancer cell apoptosis by targeting the electron transport chain. Moreover, when IR-780 combined with HBO, it exhibited effective antitumor activity by promoting cancer cell uptake of IR-780 and inducing excessive mitochondrial ROS production (as illustrated in Scheme 1). This discovery potentially offers a novel treatment paradigm for human bladder cancer.

\section{Author Contributions}

LWB\& SCM conceived and designed the experiments, supervised the experiments and revised the manuscript. SCX performed the experiments, analysed the data and drafted the manuscript. YSF and WJW took part in the animal experiments and analysis of optical properties. DLY and LJJ participated in Flow Cytometerand western blot analysis. FQ and ZY contributed to collecting humantissues. All authors have read and approved the final manuscript.

\section{References}

A R, A V, I B, H W, KM Z, D S, et al. (2013). Overcoming intrinsic multidrug resistance in melanoma by blocking the mitochondrial respiratory chain of slow-cycling JARID1B(high) cells. Cancer cell 23: 811-825.

Alfred Witjes J, Lebret T, Comperat EM, Cowan NC, De Santis M, Bruins HM, et al. (2017). Updated 2016 EAU Guidelines on Muscle-invasive and Metastatic Bladder Cancer. Eur Urol 71: 462-475.

Berkenblit A, Eder JP, Jr., Ryan DP, Seiden MV, Tatsuta N, Sherman ML, et al. (2007). Phase I clinical trial of STA-4783 in combination with paclitaxel in patients with refractory solid tumors. Clin Cancer Res 13: $584-590$.

Cao H, Zou L, He B, Zeng L, Huang Y, Yu H, et al. (2017). Albumin Biomimetic Nanocorona Improves Tumor Targeting and Penetration for Synergistic Therapy of Metastatic Breast Cancer. Advanced Functional Materials 27.

Cierlitza M, Chauvistre H, Bogeski I, Zhang X, Hauschild A, Herlyn M, et al. (2015). Mitochondrial oxidative stress as a novel therapeutic target to overcome intrinsic drug resistance in melanoma cell subpopulations. 
Experimental dermatology 24: 155-157.

Cui Q, Wang J-Q, Assaraf YG, Ren L, Gupta P, Wei L, et al.(2018). Modulating ROS to overcome multidrug resistance in cancer. Drug Resistance Updates 41: 1-25.

Davis S, Weiss M, Wong J, Lampidis T, \& Chen L (1985). Mitochondrial and plasma membrane potentials cause unusual accumulation and retention of rhodamine 123 by human breast adenocarcinoma-derived MCF7 cells. The Journal of biological chemistry 260: 13844-13850.

Deribe YL, Sun Y, Terranova C, Khan F, Martinez-Ledesma J, Gay J, et al. (2018). Author Correction: Mutations in the SWI/SNF complex induce a targetable dependence on oxidative phosphorylation in lung cancer. Nat Med 24: 1627.

Desai N (2012). Challenges in development of nanoparticle-based therapeutics. AAPS J 14: 282-295.

Dewhirst M, Mowery Y, Mitchell J, Cherukuri M, \& Secomb T (2019). Rationale for hypoxia assessment and amelioration for precision therapy and immunotherapy studies. The Journal of clinical investigation 129: 489-491.

Hao W, Chang CP, Tsao CC, \& Xu J (2010). Oligomycin-induced bioenergetic adaptation in cancer cells with heterogeneous bioenergetic organization. J Biol Chem 285: 12647-12654.

Hoskin P, Sibtain A, Daley F, \& Wilson G (2003). GLUT1 and CAIX as intrinsic markers of hypoxia in bladder cancer: relationship with vascularity and proliferation as predictors of outcome of ARCON. British journal of cancer 89: 1290-1297.

Housman G, Byler S, Heerboth S, Lapinska K, Longacre M, Snyder N, et al. (2014). Drug Resistance in Cancer: An Overview. Cancers 6: 1769-1792.

Idris NM, Jayakumar MK, Bansal A, \& Zhang Y (2015). Upconversion nanoparticles as versatile light nanotransducers for photoactivation applications. Chem Soc Rev 44: 1449-1478.

Jose C, Bellance N, \& Rossignol R (2011). Choosing between glycolysis and oxidative phosphorylation: a tumor's dilemma? Biochim Biophys Acta 1807: 552-561.

JR M, Y S, M P, S G, M B, C B, et al. (2018). An inhibitor of oxidative phosphorylation exploits cancer vulnerability. Nature medicine 24: 1036-1046.

Klyubin I, Kirpichnikova K, Ischenko A, Zhakhov A, \& Gamaley I (2000). The role of reactive oxygen species in membrane potential changes in macrophages and astrocytes. Membrane \& cell biology 13:557-566.

Kuang Y, Zhang K, Cao Y, Chen X, Wang K, Liu M, et al. (2017). Hydrophobic IR-780 Dye Encapsulated in cRGD-Conjugated Solid Lipid Nanoparticles for NIR Imaging-Guided Photothermal Therapy. ACS Appl Mater Interfaces 9: 12217-12226.

Lucky SS, Muhammad Idris N, Li Z, Huang K, Soo KC, \& Zhang Y (2015). Titania coated upconversion nanoparticles for near-infrared light triggered photodynamic therapy. ACS Nano 9: 191-205.

Luo S, Tan X, Fang S, Wang Y, Liu T, Wang X, et al. (2016). Mitochondria-Targeted Small-Molecule Fluorophores for Dual Modal Cancer Phototherapy. Advanced Functional Materials 26: 2826-2835.

Ploeg M, Aben KKH, \& Kiemeney LA (2009). The present and future burden of urinary bladder cancer in the world. World Journal of Urology 27: 289-293.

Porporato PE, Filigheddu N, Pedro JMB-S, Kroemer G, \& Galluzzi L (2017). Mitochondrial metabolism and cancer. Cell Research 28:265-280.

Roesch A, Vultur A, Bogeski I, Wang H, Zimmermann KM, Speicher D, et al. (2013). Overcoming intrinsic multidrug resistance in melanoma by blocking the mitochondrial respiratory chain of slow-cycling JARID1B(high) cells. Cancer Cell 23: 811-825. 
Sabharwal SS, \& Schumacker PT (2014). Mitochondrial ROS in cancer: initiators, amplifiers or an Achilles' heel? Nat Rev Cancer 14:709-721.

Santos J, Blackman RK, Cheung-Ong K, Gebbia M, Proia DA, He S, et al. (2012). Mitochondrial Electron Transport Is the Cellular Target of the Oncology Drug Elesclomol. PLoS ONE 7.

Selvendiran K, Kuppusamy ML, Ahmed S, Bratasz A, Meenakshisundaram G, Rivera BK, et al. (2010). Oxygenation inhibits ovarian tumor growth by downregulating STAT3 and cyclin-D1 expressions. Cancer Biol Ther 10: 386-390.

Stepień K, Ostrowski RP, \& Matyja E (2016). Hyperbaric oxygen as an adjunctive therapy in treatment of malignancies, including brain tumours. Medical Oncology 33.

Svatek RS, Hollenbeck BK, Holmang S, Lee R, Kim SP, Stenzl A, et al. (2014). The economics of bladder cancer: costs and considerations of caring for this disease. Eur Urol 66: 253-262.

Tan X, Luo S, Long L, Wang Y, Wang D, Fang S, et al. (2017). Structure-Guided Design and Synthesis of a Mitochondria-Targeting Near-Infrared Fluorophore with Multimodal Therapeutic Activities. Advanced Materials 29.

Uthaman S, Mathew AP, Park HJ, Lee BI, Kim HS, Huh KM, et al.(2018). IR 780-loaded hyaluronic acid micelles for enhanced tumor-targeted photothermal therapy. Carbohydr Polym 181: 1-9.

Wang Y, Liu T, Zhang E, Luo S, Tan X, \& Shi C (2014). Preferential accumulation of the near infrared heptamethine dye IR-780 in the mitochondria of drug-resistant lung cancer cells. Biomaterials 35: 4116-4124.

Wang Y, Luo S, Zhang C, Liao X, Liu T, Jiang Z, et al. (2018). An NIR-Fluorophore-Based Therapeutic Endoplasmic Reticulum Stress Inducer. Adv Mater: e1800475.

Weinberg SE, \& Chandel NS (2015). Targeting mitochondria metabolism for cancer therapy. Nat Chem Biol 11: 9-15.

Wen S, Zhu D, \& Huang P (2013). Targeting cancer cell mitochondria as a therapeutic approach. Future medicinal chemistry 5: 53-67.

Wu L, Zhao J, Cao K, Liu X, Cai H, Wang J, et al. (2018). Oxidative phosphorylation activation is an important characteristic of DOX resistance in hepatocellular carcinoma cells. Cell Communication and Signaling 16.

WX Z, JD R, \& E W (2016). Mitochondria and Cancer. Molecular cell 61: 667-676.

Xiao T, Fan JK, Huang HL, Gu JF, Li LY, \& Liu XY (2010). VEGI-armed oncolytic adenovirus inhibits tumor neovascularization and directly induces mitochondria-mediated cancer cell apoptosis. Cell Res 20: 367-378.

Y S, SK L, Q L, SV I, HY W, Z W, et al. (2019). Gboxin is an oxidative phosphorylation inhibitor that targets glioblastoma. Nature 567: 341-346.

Yan F, Duan W, Li Y, Wu H, Zhou Y, Pan M, et al. (2016). NIR-Laser-Controlled Drug Release from DOX/IR-780-Loaded Temperature-Sensitive-Liposomes for Chemo-Photothermal Synergistic Tumor Therapy. Theranostics 6: 2337-2351.

Zehnder P, Studer UE, Skinner EC, Thalmann GN, Miranda G, Roth B, et al. (2013). Unaltered oncological outcomes of radical cystectomy with extended lymphadenectomy over three decades. BJU Int 112:E51-58.

Zhang C, Liu T, Su Y, Luo S, Zhu Y, Tan X, et al. (2010). A near-infrared fluorescent heptamethine indocyanine dye with preferential tumor accumulation for in vivo imaging. Biomaterials 31:6612-6617.

Zhang E, Luo S, Tan X, \& Shi C (2014). Mechanistic study of IR-780 dye as a potential tumor targeting and drug delivery agent. Biomaterials 35: 771-778. 
Zielonka J, Joseph J, Sikora A, Hardy M, Ouari O, Vasquez-Vivar J, et al. (2017). Mitochondria-Targeted Triphenylphosphonium-Based Compounds: Syntheses, Mechanisms of Action, and Therapeutic and Diagnostic Applications. Chemical reviews 117: 10043-10120.

\section{Figure legends}

Figure 1. IR-780 specifically targets tumor tissue and locates to cancer cell mitochondria. (A) Nude mice with T24 subcutaneous tumor xenografts and C57 BL/6 mice with MB49 subcutaneous transplanted tumors were subjected to a single-dose intraperitoneal administration of IR-780 at $1 \mathrm{mg} / \mathrm{kg}(\mathrm{n}=5)$. The dissected organs at $24 \mathrm{~h}$ after injection were subjected to near-infrared fluorescence (NIRF) imaging. (B) The fluorescence intensity of dissected organs and tumors in (A). (C) Tumor and the normal tissue from patients with MIBC were subjected to NIRF imaging after incubation with IR-780 at $10 \mu \mathrm{M}$ for $2 \mathrm{~h}(\mathrm{n}=5)$. (D) The fluorescence intensity of (C). (D) Colocalization of IR-780 with a mitochondria-specific tracker (MitoTracker Green) in T24 and MB49 bladder cancer cell lines was imaged using a confocal microscope; the HK2 and SV-HUC-1 normal cell lines were used as controls. The nuclei were stained with Hoechst 33258. Scale bar $\left.=25 \mu \mathrm{m} . \quad{ }^{*}, \mathrm{p}<0.05\right)$.

Figure 2 . The anti-tumor effect of IR-780 and influence on the mitochondrial activities of T24 cells. (A) T24, 5637, TCCSUP, and MB49 bladder cancer cell viability were measured using a CCK-8 kit after treatment with various doses of IR-780 for $48 \mathrm{~h}$. (B) T24 cells were treated with IR-780 for $24 \mathrm{~h}$ and harvested for detection of apoptosis using flow cytometry. (C) Mitochondrial ROS levels, (D) ATP production, (E) mitochondrial membrane potential, and (F) Mitochondrial complex I activity were detected after treatment with IR-780 for $24 \mathrm{~h}$. (G) Western blot detection of recombinant human DNUFS1 protein treated with IR-780 in vitro. $(*, p<0.05)$.

Figure 3 . HBO promotes IR-780 antitumor efficacy in vitro. (A) Different bladder cancer cell viability was tested after treatment with IR-780 with or without HBO for $48 \mathrm{~h}$. (B) Image of colony formation by T24 cells after treatment with 0 or $7.5 \mu \mathrm{M}$ IR-780 with or without HBO for $6 \mathrm{~h}$ before seeding. (C) The colony formation efficiency of T24 cells at 14 days after seeding. (D) T24 cells were treated with 0 or $7.5 \mu \mathrm{M}$ IR-780 with or without HBO for $24 \mathrm{~h}$ and harvested for detection of apoptosis using flow cytometry. (E) Analysis of variance statistics of the cytometry data derived from samples presented in (D). (F) The morphology of T24 cells after treatment with 0 or $7.5 \mu \mathrm{M}$ IR-780 combined or not with HBO was observed by inverted microscopy. Scale bar $=25 \mu \mathrm{m}$. (G) Expression of apoptosis-related proteins in T24 cells. Cleaved PARP, caspase-3, c-caspase-9, and cytochrome c levels were tested by western blotting. (H) Viability of T24 cells treated with IR-780+HBO in the presence of Z-VAD (an apoptosis inhibitor). (*, $\mathrm{p}<0.05$ ).

Figure 4 . The antitumor efficacy of IR-780+HBO in vivo. Treatment was carried out by intraperitoneal injection every two days for a total of 5 treatments. The IR-780+HBO and IR-780 groups were treated with $3 \mathrm{mg} / \mathrm{kg}$ IR-780. (A) The transplanted tumor size was monitored every 3 days using a sliding caliper. After excision from the mice, the transplanted tumor were photographed (B) and weighed (C). Scale bar $=1 \mathrm{~cm}$. (D) The body weights of mice were measured every 3 days before excision. (E) Long-term observation of tumor volume after mice were treated with IR-780+HBO or DDP. Images of the mouse transplanted tumor at day 28 are shown in $(F)$. Scale bar $=1 \mathrm{~cm}$. (G) H\&E staining in major organs of mice. Scale bar $=50$ $\left.\mu \mathrm{m} .{ }^{*}, \mathrm{p}<0.05\right)$.

Figure 5 . HBO promotes uptake of IR-780 in cancer cells by increasing the plasma membrane potential. T24 cells were treated with the indicated doses of IR-780 with or without HBO and imaged using a fluorescence microscope (A), and fluorescence intensity was measured using flow cytometry (B) immediately after the completion of HBO. Scale bar $=25 \mu \mathrm{m}$. (C) The fluorescence spectrum of $7.5 \mu \mathrm{M}$ IR-780 in cell culture medium combined or not with $\mathrm{HBO}$ with $740 \mathrm{~nm}$ using an excitation wavelength from $750^{\sim} 900 \mathrm{~nm}$ to scan their emission spectra. (D) T24 cells were treated with 0 or $7.5 \mu \mathrm{M}$ IR-780 with or without HBO for 24 $\mathrm{h}$, and NDUFS1 was detected by western blotting. (E) T24 cells were treated with pre-IR-780 (IR-780 was removed after incubation for 30 minutes) $+\mathrm{HBO}$ and stained with DiBAC4 to detect the plasma membrane potential by flow cytometry. (F) Plasma membrane potential was tested after cell incubation different doses 
of $\mathrm{K}^{+}$medium for $1 \mathrm{~h}$. Cells were pre-incubated for $1 \mathrm{~h}$ with the indicated doses of $\mathrm{K}^{+}$medium followed by $2 \mu \mathrm{M}$ IR-780 for $10 \mathrm{~min}$, and the fluorescence intensity of IR-780 was examined (G, H). Scale bar $=50 \mu \mathrm{m}$. After $2 \mu \mathrm{M}$ IR-780 treatment for 30min, the cells were incubated with the indicated doses of $\mathrm{K}^{+}$medium and treated with HBO, and the fluorescence intensity of IR-780 was examined (I). (J) T24 cells were treated with IR-780, pre-IR-780 + HBO, or IR-780+HBO, and cell viability was measured. (*, p<0.05).

Figure 6 . IR-780+HBO induces excessive mitochondrial ROS production. (A) Fluorescence image of the ROS probe DCFDA by T24 cells after treatment with 0 or $7.5 \mu \mathrm{M}$ IR-780 with or without HBO. Scale bar $=100 \mu \mathrm{m}$. (B) DCFDA fluorescence intensity was measured using flow cytometry. (C) Fluorescence images of T24 cells labeled with DCFDA and mitoSOX after treatment with IR-780+HBO. Scale bar $=25 \mu \mathrm{m}$. Mitochondrial ROS production detected using mitoSOX (D). (E) IR-780+HBO-induced cellular mitochondrial ROS levels were positively correlated with cell death. (F) Mitochondrial ROS levels were detected after treatment with IR-780+HBO in the presence of DPI (an NADPH inhibitor), MitoQ (a mitochondrial ROS inhibitor) or NAC, and then cell viability was detected $(\mathrm{G})$. (H) Western blot detection of apoptosis induction by IR-780+HBO in the presence or absence of MitoQ or NAC. T24 cells were treated with $7.5 \mu \mathrm{M}$ IR-780 with or without the indicated concentrations of $\mathrm{H}_{2} \mathrm{O}_{2}$ for $24 \mathrm{~h}$ and then harvested for detection of apoptosis using flow cytometry (I). Mitochondrial ROS levels of T24 cells were measured after treatment with IR-780+HBO in the present of $21 \% \mathrm{O}_{2}, 50 \% \mathrm{O}_{2}, 100 \% \mathrm{O}_{2}(\mathrm{~J})$ or TTFA $(\mathrm{K})$. (L) Detection of mitochondrial membrane potential induced by IR-780+HBO. Mitochondrial morphology in T24 cells after treatment with 0 or $7.5 \mu \mathrm{M}$ IR-780 combined or not with HBO was observed by transmission electron microscopy (M). Scale bar $=1 \mu \mathrm{m} .(*, \mathrm{p}<0.05)$.

Figure 7. The effects of IR-780+HBO on DDP-resistant T24 cells. (A) Staining of IR-780 in T24 and T24/DDP cancer cells was imaged using a fluorescence microscope, and the fluorescence intensity was determined by flow cytometry (B). Scale bar $=50 \mu \mathrm{m}$. (C) NIRF imaging of T24/DDP subcutaneous tumor xenografts using IR-780. (D) Viability of T24 cells and T24/DDP cells treated with DDP. (E) Cell viability of T24/DDP cells treated with IR-780, IR-780+HBO or DDP. (F) The volumes of T24/DDP cell tumors were observed after nude mice were treated with IR-780+HBO or DDP. Images of the xenografts after excision from the mice at day 19 are shown in $(\mathrm{G})$. Scale bar $=1 \mathrm{~cm}$. (H) In the IR-780+HBO treatment group, the T24/DDP tumor xenografts appeared to be ruptured. $(*, \mathrm{p}<0.05)$.

Figure 8 . Proposed model of mitochondrial apoptosis-inducing mechanism by IR-780+HBO. IR-780 preferentially accumulates in the mitochondria of tumor cells by targeting mitochondrial protein NDUSF1, causing many electrons to leak out; combination with HBO could induce cancer cell apoptosis by promoting cancer cell uptake of IR-780 and inducing excessive mitochondrial ROS production.

\section{Hosted file}

Figure.pdf available at https://authorea.com/users/371058/articles/489535-hyperbaric-oxygenenhanced-mitochondria-targeted-chemotherapy-in-bladder-cancer 